\title{
Blow-up criterion of classical solutions for the incompressible nematic liquid crystal flows
}

\author{
Zhensheng Gao ${ }^{\text {a }}$ \\ School of Mathematical Sciences, Huaqiao University, Quanzhou 362021, China. \\ Zhong Tan \\ School of Mathematical Sciences, Xiamen University, Xiamen 361005, China
}

\begin{abstract}
In this paper, we consider the short time classical solution to a simplified hydrodynamic flow modeling incompressible, nematic liquid crystal materials in $\mathbb{R}^{3}$. We establish a criterion for possible breakdown of such solutions at a finite time. More precisely, if $(u, d)$ is smooth up to time $T$ provided that $\int_{0}^{T}\|\nabla \times u(t, \cdot)\|_{B M O\left(\mathbb{R}^{3}\right)}+\|\nabla d(t, \cdot)\|_{L^{4}\left(\mathbb{R}^{3}\right)}^{8} d t<\infty$.
\end{abstract}

Keywords: Incompressible nematic liquid crystal; Blow up criterion.

2000 Mathematics Subject Classification: 76W05, 35Q35, 35D05.

\section{Introduction}

We consider the following hydrodynamic system modeling the flow of liquid crystal materials in $\mathbb{R}^{3}$ (see $[2,3,13,17]$ ) and references therein):

$$
\left\{\begin{array}{l}
u_{t}+u \cdot \nabla u+\nabla P=\mu \Delta u-\nabla d \cdot \Delta d \\
\partial_{t} d+u \cdot \nabla d=\Delta d+|\nabla d|^{2} d \\
\nabla \cdot u=0, \quad|d|=1
\end{array}\right.
$$

for $(t, x) \in[0,+\infty) \times \mathbb{R}^{3}$. Here $u: \mathbb{R}^{3} \rightarrow \mathbb{R}^{3}$ represents the velocity field of the incompressible viscous fluid, $\mu>0$ is the coefficient of viscosity. $P: \mathbb{R}^{3} \rightarrow \mathbb{R}$ represents the pressure function, $d: \mathbb{R}^{3} \rightarrow \mathbb{S}^{2}$ represents the macroscopic average of the nematic liquid crystal orientation field. In this paper, we are interested in the Cauchy problem (1.1) with the initial value

$$
(u(x, 0), d(x, 0))=\left(u_{0}(x), d_{0}(x)\right)
$$

satisfying the following compatibility condition:

$$
\nabla \cdot u_{0}(x)=0, \quad\left|d_{0}(x)\right|=1, \lim _{|x| \rightarrow \infty} d_{0}(x)=a \in \mathbb{S}^{2},
$$

where $a$ is a given unit vector.

The above system is a simplified version of the Ericksen-Leslie model, which reduces to the Ossen-Frank model in the static case, for the hydrodynamics of nematic liquid crystals developed during the period of 1958 through 1968 [2, 3, 13]. It is a macroscopic continuum description of the time evolution of the materials under the influence of both the flow field $u(x, t)$, the macroscopic description of the microscopic orientation configurations $d(x, t)$ of rod-like liquid crystals. Roughly speaking, the system (1.1) is a coupling between the non-homogeneous

${ }^{\text {a} E-m a i l ~ a d d r e s s: ~ g a o z h e n s h e n g @ h q u . e d u . c n . ~}$ 
Navier-Stokes equation and the transported flow harmonic maps. Due to the physical importance and mathematical challenges, the study on nematic liquid crystals has attracted many physicists and mathematicians, the readers are referred to $[4,5,6,7,8,9,12,14,15,16,17$, $18,19,20,21,23]$ and references therein. The mathematical analysis of the liquid crystal flows was initiated by Lin [16], Lin and Liu in [17, 18]. For any bounded smooth domain in $\mathbb{R}^{2}$, Lin , Lin and Wang [18] have proved the global existence of Leray-Hopf type weak solutions to system (1.1) which are smooth everywhere except on finitely many time slices (see [5] for the whole space). The uniqueness of weak solutions in two dimension was studied by [12, 20, 23]. Hong and Xin [6] studied the global existence for general Ericksen-Leslie system in dimension two. However, the global existence of weak solutions to the incompressible nematic liquid crystal flow equation (1.1) in three dimension with large initial data is still an outstanding open question. Huang et al. [9]constructed two examples of finite time singularity. Lin-Wang [21] established establish the global existence of a weak solution under geometric angle condition. For arbitrary data, the global existence of weak solution is still an open problem.

The local well-posedness of the Cauchy problem of system (1.1) is rather standard (see $[5,8,19])$. At present, there is no global-in-time existence theory for classical solutions to system (1.1). In this paper, we will consider the short time classical solution to (1.1)-(1.2) and some criterion that characterizes the first finite singular time. Motivated by the famous work [1], Huang and Wang [8] have obtained a BKM type blow-up criterion. The main subject of this paper is inspired [11], we establish a new blow up criterion. The techniques involved in this paper are much different from [8], which we believe that the result may have its own interest. Our main results are formulated as the following theorem:

Theorem 1.1. Let $\mu=0, u_{0} \in H^{\ell}\left(\mathbb{R}^{3}\right)$ with $\nabla \cdot u_{0}=0$ and $d_{0}-a \in H^{\ell+1}\left(\mathbb{R}^{3}\right)$ with $\left|d_{0}\right|=1$ for $\ell \geq 3$. Suppose that $(u, d)$ is a smooth solution to the system (1.1)-(1.2), then for given $T>0$, $(u, d)$ is smooth up to time $T$ provided that

$$
\int_{0}^{T}\|\nabla \times u(t, \cdot)\|_{B M O\left(\mathbb{R}^{3}\right)}+\|\nabla d(t, \cdot)\|_{L^{4}\left(\mathbb{R}^{3}\right)}^{8} d t<\infty .
$$

Notations. We denote by $L^{p}, W^{m, p}$ the usual Lebesgue and Sobolev spaces on $\mathbb{R}^{3}$ and $H^{m}=W^{m, 2}$, with norms $\|\cdot\|_{L^{p}},\|\cdot\|_{W^{m, p}}$ and $\|\cdot\|_{H^{m}}$ respectively. For the sake of conciseness, we do not distinguish functional space when scalar-valued or vector-valued functions are involved. We denote $\nabla=\partial_{x}=\left(\partial_{1}, \partial_{2}, \partial_{3}\right)$, where $\partial_{i}=\partial_{x_{i}}, \nabla_{i}=\partial_{i}$ and put $\partial_{x}^{l} f=\nabla^{l} f=\nabla\left(\nabla^{l-1} f\right)$. We assume $C$ be a positive generic constant throughout this paper that may vary at different places and the integration domain $\mathbb{R}^{3}$ will be always omitted without any ambiguity. Finally, $\langle\cdot, \cdot\rangle$ denotes the inner-product in $L^{2}\left(\mathbb{R}^{3}\right)$. $\|(a, b)\|_{W^{m, p}}$ denotes $\|a\|_{W^{m, p}}+\|b\|_{W^{m, p}}$.

Remark 1.1. It is standard that the condition (1.3) is preserved by the flow. In fact, first notice that the divergence free of the velocity field $u$ can be justified by the initial assumption that $\nabla \cdot u=0$. Moreover, applying the maximum principle to the equation for $|d|^{2}$, one also can easily see that $|d|=1$ under the initial assumption that $\left|d_{0}\right|=1$.

\section{Proof of Theorem 1.1}

We prove our theorem in this section. The first bright idea to reduce many complicated computations lies in that we just need to do the lowest order and highest order energy estimates for the solutions. This is motivated by the following observation:

$$
\|f\|_{H^{\ell}}^{2} \leq C\left\|\left(f, \nabla^{\ell} f\right)\right\|_{L^{2}}^{2}, \quad \forall f \in H^{\ell} .
$$


The above inequality can be easily established in light of Young's inequality and GagliardoNirenberg's inequality

$$
\left\|\nabla^{i} f\right\|_{L^{p}} \leq C(p)\|f\|_{L^{q}}^{\alpha}\left\|\nabla^{\ell} f\right\|_{L^{r}}^{1-\alpha}, \quad \forall f \in H^{\ell}
$$

where $\frac{1}{p}-\frac{i}{3}=\frac{1}{q} \alpha+\left(\frac{1}{r}-\frac{\ell}{3}\right)(1-\alpha)$ with $i \leq \ell$.

As in [11], the following logarithmic Sobolev's plays an important role in our proofs, which is proved in [10] and is an improved version of that in [1].

Lemma 2.1. Let $f \in W^{2, p}\left(\mathbb{R}^{3}\right)$ with $p>3$, then the following logarithmic Sobolev's embedding theorem holds for all divergence free vector fields $f$ :

$$
\|\nabla f\|_{L^{\infty}} \leq C\left[1+\|f\|_{L^{2}}+\|\nabla \times f\|_{B M O} \ln \left(1+\|f\|_{W^{2, p}}\right)\right] .
$$

Now we are in a position to prove our Theorem 1.1.

Proof of Theorem 1.1 First of all, for classical solutions to (1.1)-(1.2), one has the following basic energy law:

$$
\|u(t)\|_{L^{2}}^{2}+\|\nabla d(t)\|_{L^{2}}^{2}+2 \int_{0}^{t}\|u(s)\|_{L^{2}}^{2}+\left\|\Delta d(s)+|\nabla d|^{2} d(s)\right\|_{L^{2}}^{2} d s=\left\|u_{0}\right\|_{L^{2}}^{2}+\left\|\nabla d_{0}\right\|_{L^{2}}^{2} \quad \forall t>0
$$

Let's concentrate on the case $\ell=3$. For each multi-index $\alpha$ with $|\alpha| \leq 3$, by applying $\partial_{x}^{\alpha}$ to $(1.1)_{1}$ and $\partial_{x}^{\alpha+1}$ to $(1.1)_{2}$, multiplying them by $\partial_{x}^{\alpha} u, \partial_{x}^{\alpha+1} d$ respectively and then integrating them over $\mathbb{R}^{3}$, we arrive at

$$
\begin{aligned}
& \frac{1}{2} \frac{d}{d t}\left\|\partial_{x}^{\alpha}(u, \nabla d)(t)\right\|_{L^{2}}^{2}+\left\|\partial_{x}^{\alpha} \nabla u(t)\right\|_{L^{2}}^{2}+\left\|\partial_{x}^{\alpha} \Delta d(t)\right\|_{L^{2}}^{2} \\
= & -\left\langle\partial_{x}^{\alpha}(u \cdot \nabla u)(t), \partial_{x}^{\alpha} u(t)\right\rangle-\left\langle\partial_{x}^{\alpha}(\Delta d \cdot \nabla d)(t), \partial_{x}^{\alpha} u(t)\right\rangle \\
& -\left\langle\partial_{x}^{\alpha+1}(u \cdot \nabla d)(t), \partial_{x}^{\alpha+1} d(t)\right\rangle+\left\langle\partial_{x}^{\alpha+1}\left(|\nabla d|^{2} d\right)(t), \partial_{x}^{\alpha+1} d(t)\right\rangle \\
= & \sum_{i=1}^{4} I_{|\alpha|, i}
\end{aligned}
$$

where $I_{|\alpha|, i}$ are the corresponding terms in the above equation which will be estimated as following:

For $|\alpha|=1$ in (2.5), integrating by parts and using the divergence free condition $\nabla \cdot u=0$, we have

$$
\left|I_{1,1}\right|=\left|\left\langle\partial_{x}^{1}(u \cdot \nabla u)(t), \partial_{x}^{1} u(t)\right\rangle\right|=\left|\left\langle\partial_{x}^{1} u(t) \cdot \nabla u(t), \partial_{x}^{1} u(t)\right\rangle\right| \leq C\|\nabla u(t)\|_{L^{\infty}}\|\nabla u(t)\|_{L^{2}}^{2} .
$$

Combining Cauchy's inequality, Sobolev's inequality and the fact $|\nabla d|^{2}=-d \cdot \Delta d$ (since $\left.|d|=1\right)$ gives

$$
\begin{aligned}
\left|I_{1,2}\right| & =\left|\left\langle\partial_{x}^{1}(\Delta d \cdot \nabla d)(t), \partial_{x}^{1} u(t)\right\rangle\right|=\left|\left\langle\Delta d(t) \cdot \nabla d(t), \partial_{x}^{2} u(t)\right\rangle\right| \\
& \leq C\|\Delta d(t)\|_{L^{3}}^{3}+\frac{1}{8}\left\|\nabla^{2} u(t)\right\|_{L^{2}}^{2} \\
& \left.\leq C\|\nabla d(t)\|_{L^{6}}^{\frac{6}{5}}\|\nabla \Delta d(t)\|_{L^{2}}^{\frac{9}{5}}+\frac{1}{8}\left\|\nabla^{2} u(t)\right\|_{L^{2}}^{2}\right) \\
& \left.\leq C\|\nabla d(t)\|_{L^{4}}^{12}+\frac{1}{8}\left(\left\|\nabla^{2} u(t)\right\|_{L^{2}}^{2}\right)+\|\nabla \Delta d(t)\|_{L^{2}}^{2}\right) .
\end{aligned}
$$

Similarly,

$$
\begin{aligned}
\left|I_{1,3}\right|= & \left|\left\langle\partial_{x}^{2} u(t) \cdot \nabla d(t)+\partial_{x} u(t) \cdot \nabla \partial_{x} d(t), \partial_{x}^{2} d(t)\right\rangle\right| \\
\leq & C\left(\|\nabla u(t)\|_{L^{\infty}}\left\|\nabla^{2} d(t)\right\|_{L^{2}}^{2}+\|\nabla d(t)\|_{L^{4}}^{12}\right)+\frac{1}{8}\left(\left\|\nabla^{2} u(t)\right\|_{L^{2}}^{2}+\|\nabla \Delta d(t)\|_{L^{2}}^{2}\right), \\
& \left|I_{1,4}\right|=\left|\left\langle\partial_{x}^{1}\left(|\nabla d|^{2} d\right)(t), \partial_{x} \Delta d(t)\right\rangle\right| \leq C\|\nabla d(t)\|_{L^{4}}^{12}+\frac{1}{8}\|\nabla \Delta d(t)\|_{L^{2}}^{2} .
\end{aligned}
$$


Taking the above estimates in (2.5) for $|\alpha|=1$, we arrive at

$$
\begin{aligned}
& \frac{d}{d d}\left\|\left(\nabla u, \nabla^{2} d\right)(t)\right\|_{L^{2}}^{2}+\left\|\left(\nabla^{2} u, \nabla^{3} d\right)(t)\right\|_{L^{2}}^{2} \\
\leq & C\left[\|\nabla u(t)\|_{L^{\infty}}\left(\|\nabla u(t)\|_{L^{2}}^{2}+\left\|\nabla^{2} d(t)\right\|_{L^{2}}^{2}\right)+\|\nabla d(t)\|_{L^{4}}^{12}\right] .
\end{aligned}
$$

Next we derive an estimate for $\|\nabla d\|_{L^{4}}^{4}$. Differentiating $(1.1)_{2}$ with respect to $x$, we have

$$
\partial_{x} d_{t}-\Delta \partial_{x} d=\partial_{x}\left(|\nabla d|^{2} d-u \cdot \nabla d\right)
$$

Multiplying (2.7) by $|\nabla d|^{2} \partial_{x} d$ and integrating over $\mathbb{R}^{3}$, we have

$$
\begin{aligned}
& \frac{1}{4} \frac{d}{d t}\|\nabla d(t)\|_{L^{4}}^{4}+\|\mid\| \nabla d(t)\left\|\nabla^{2} d(t)\right\|\left\|_{L^{2}}^{2}+\frac{1}{2}\right\| \partial_{x}\left(|\nabla d(t)|^{2}\right) \|_{L^{2}}^{2} \\
= & \left\langle|\nabla d(t)|^{2} \partial_{x} d(t), \partial_{x}\left(|\nabla d(t)|^{2} d-u \cdot \nabla d(t)\right)\right\rangle \\
= & \left.\|\nabla d(t)\|_{L^{6}}^{6}+\left\langle|\nabla d(t)|^{2} \partial_{x} d(t), 2 \nabla d(t) \nabla \partial_{x} d(t) d(t)-\partial_{x} u(t) \cdot \nabla d(t)\right)\right\rangle \\
\leq & C\left(\|\nabla d(t)\|_{L^{3}}^{3}+\|\nabla u(t)\|_{L^{\infty}}\|\nabla d(t)\|_{L^{4}}^{4}\right) \\
\leq & C\left(\|\nabla u(t)\|_{L^{\infty}}\|\nabla d(t)\|_{L^{4}}^{4}+\|\nabla d(t)\|_{L^{4}}^{12}\right)+\frac{1}{8}\left\|\nabla^{3} d(t)\right\|_{L^{2}}^{2} .
\end{aligned}
$$

Adding the above inequality to (2.6), we arrive at

$$
\begin{aligned}
& \frac{d}{d t}\left(\left\|\left(\nabla u, \nabla^{2} d\right)(t)\right\|_{L^{2}}^{2}+\|\nabla d(t)\|_{L^{4}}^{4}\right)+\left\|\left(\nabla^{2} u(t), \nabla^{3} d(t),\left|\nabla d(t) \| \nabla^{2} d(t)\right|\right)\right\|_{L^{2}}^{2} \\
\leq & C\left(\|\nabla u(t)\|_{L^{\infty}}+\|\nabla d(t)\|_{L^{4}}^{8}\right)\left(\left\|\left(\nabla u, \nabla^{2} d\right)(t)\right\|_{L^{2}}^{2}+\|\nabla d(t)\|_{L^{4}}^{4}\right),
\end{aligned}
$$

which gives that

$$
\begin{aligned}
& \left\|\left(\nabla u, \nabla^{2} d\right)(t)\right\|_{L^{2}}^{2}+\|\nabla d(t)\|_{L^{4}}^{4}+\int_{t_{0}}^{t}\left\|\left(\nabla^{2} u, \nabla^{3} d,\left|\nabla d \| \nabla^{2} d\right|\right)(s)\right\|_{L^{2}}^{2} d s \\
\leq & C e^{C \int_{t_{0}}^{t}\left(\|\nabla u(s)\|_{L^{\infty}+\| \nabla d(s)}^{8} \|_{L^{4}}^{4} d s\right.}\left(\left\|\left(\nabla u, \nabla^{2} d\right)\left(t_{0}\right)\right\|_{L^{2}}^{2}+\left\|\nabla d\left(t_{0}\right)\right\|_{L^{4}}^{4}\right) .
\end{aligned}
$$

Noting the condition (1.4) in Theorem 1.1, thus for any fixed small constant $\varepsilon>0$, there exists $T_{*}(\varepsilon)<T$ such that

$$
\int_{T_{*}}^{T}\left(\|\nabla \times u\|_{B M O}+\|\nabla d\|_{L^{4}}^{8}\right)(s) d s \leq \varepsilon .
$$

Define the temporal energy functional:

$$
H(t)=\sup _{T_{*} \leq s \leq t}\left(\left\|\nabla^{3} u(s)\right\|_{L^{2}}^{2}+\left\|\nabla^{4} d(s)\right\|_{L^{2}}^{2}\right)
$$

In light of (2.1), (2.3), (2.4), (2.9) and (2.10), we arrive at

$$
\begin{aligned}
& \left\|\left(\nabla u, \nabla^{2} d\right)(t)\right\|_{L^{2}}^{2}+\|\nabla d(t)\|_{L^{4}}^{4}+\int_{T_{*}}^{t}\left\|\left(\nabla^{2} u, \nabla^{3} d,\left|\nabla d \| \nabla^{2} d\right|\right)(t)\right\|_{L^{2}}^{2} d s
\end{aligned}
$$

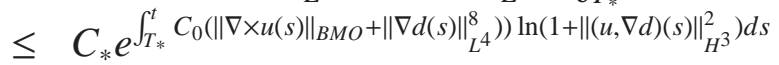

$$
\begin{aligned}
& \leq C_{*} e^{C_{0} \varepsilon \ln (1+H(t))}=C_{*}(1+H(t))^{C_{0} \varepsilon}, \quad \forall T_{*} \leq t<T,
\end{aligned}
$$

where $C_{0}$ depends on $\left\|\left(u_{0}, \nabla d_{0}\right)\right\|_{L^{2}}^{2}$ and $C_{*}$ depends on $\left\|\left(\nabla u, \nabla^{2} d\right)\right\|_{L^{2}}^{2}+\|\nabla d\|_{L^{4}}^{4}$.

Next for $|\alpha|=3$. For $I_{3,1}$, we need to use the following Moser-type inequality (see [22], p. 43):

$$
\left\|D^{s}(f g)\right\|_{L^{2}} \leq C\left(\|g\|_{L^{\infty}}\left\|\nabla^{s} f\right\|_{L^{2}}+\|f\|_{L^{\infty}}\left\|\nabla^{s} g\right\|_{L^{2}}\right) .
$$


Thus we have

$$
\begin{aligned}
\left|I_{3,1}\right| & =\left|\left\langle\partial_{x}^{2} \operatorname{div}(u(t) \otimes u(t)), \partial_{x}^{4} u(t)\right\rangle\right| \\
& \leq C\left\|\nabla^{3}(u(t) \otimes u(t))\right\|_{L^{2}}^{2}+\frac{1}{16}\left\|\nabla^{4} u(t)\right\|_{L^{2}}^{2} \\
& \leq C\|u(t)\|_{L^{\infty}}^{2}\left\|\nabla^{3} u(t)\right\|_{L^{2}}^{2}+\frac{1}{16}\left\|\nabla^{4} u(t)\right\|_{L^{2}}^{2} \\
& \leq C\left\{\|\nabla u(t)\|_{L^{2}}^{\frac{5}{6}}\left\|\nabla^{4} u(t)\right\|_{L^{2}}^{\frac{1}{6}}\|\nabla u(t)\|_{L^{2}}^{\frac{1}{3}}\left\|\nabla^{4} u(t)\right\|_{L^{2}}^{\frac{2}{3}}\right\}^{2}+\frac{1}{16}\left\|\nabla^{4} u(t)\right\|_{L^{2}}^{2} \\
& \leq C\|\nabla u(t)\|_{L^{2}}^{14}+\frac{1}{8}\left\|\nabla^{4} u(t)\right\|_{L^{2}}^{2} .
\end{aligned}
$$

For $I_{3,2}$, we apply (2.12) and the fact

$$
\Delta d \cdot \nabla d=\nabla \cdot\left(\nabla d \odot \nabla d-\frac{1}{2}|\nabla d|^{2} I\right)
$$

where $\nabla d \odot \nabla d$ denotes the symmetric $3 \times 3$ matrix: $(\nabla d \odot \nabla d)_{i j}=\nabla_{i} d \cdot \nabla_{j} d, 1 \leq i, j \leq 3$. We obtain

$$
\begin{aligned}
\left|I_{3,2}\right| & =\left|\left\langle\partial_{x}^{2} \nabla \cdot\left(\nabla d(t) \odot \nabla d(t)-\frac{1}{2}|\nabla d(t)|^{2} I\right), \partial_{x}^{4} u(t)\right\rangle\right| \\
& \leq C\left\|\nabla^{3}\left(\nabla d(t) \odot \nabla d(t)-\frac{1}{2}|\nabla d(t)|^{2} I\right)\right\|_{L^{2}}^{2}+\frac{1}{8}\left\|\nabla^{4}\right\|_{L^{2}}^{2} \\
& \leq C\|\nabla d(t)\|_{L^{\infty}}^{2}\left\|\nabla^{4} d(t)\right\|_{L^{2}}^{2}+\frac{1}{8}\left\|\nabla^{4} u(t)\right\|_{L^{2}}^{2} \\
& \leq C\left\{\left\|\nabla^{2} d(t)\right\|_{L^{2}}^{\frac{5}{6}}\left\|\nabla^{5} d(t)\right\|_{L^{2}}^{6}\left\|\nabla^{2} d(t)\right\|_{L^{2}}^{\frac{1}{3}}\left\|\nabla^{5} d(t)\right\|_{L^{2}}^{\frac{2}{3}}\right\}^{2}+\frac{1}{8}\left\|\nabla^{4} u(t)\right\|_{L^{2}}^{2} \\
& \leq C\left\|\nabla^{2} d(t)\right\|_{L^{2}}^{14}+\frac{1}{8}\left(\left\|\nabla^{4} u(t)\right\|_{L^{2}}^{2}+\left\|\nabla^{5} d(t)\right\|_{L^{2}}^{2}\right) .
\end{aligned}
$$

Similar as the proof of (2.14), $I_{3,3}, I_{3,4}$ can be bounded as follow:

$$
\begin{aligned}
\left|I_{3,3}\right|= & \left|\left\langle\partial_{x}^{3}(u \cdot \nabla d), \partial_{x}^{5} d\right\rangle\right| \\
\leq & C\left\|\partial_{x}^{3}(u \cdot \nabla d)\right\|_{L^{2}}^{2}+\frac{1}{16}\left\|\nabla^{5} d\right\|_{L^{2}}^{2} \\
\leq & C\left(\|\nabla d\|_{L^{\infty}}^{2}\left\|\nabla^{3} u\right\|_{L^{2}}^{2}+\|u\|_{L^{\infty}}^{2}\left\|\nabla^{4} u\right\|_{L^{2}}^{2}\right)+\frac{1}{16}\left\|\nabla^{4} u\right\|_{L^{2}}^{2} \\
\leq & C\left\{\left\|\nabla^{2} d\right\|_{L^{\frac{5}{6}}}^{2}\left\|\nabla^{5} d\right\|_{L^{2}}^{6}\|\nabla u\|_{L^{2}}^{\frac{1}{3}}\left\|\nabla^{4} u\right\|_{L^{2}}^{\frac{2}{3}}\right. \\
& \left.+\|\nabla u\|_{L^{2}}^{\frac{5}{6}}\left\|\nabla^{4} u\right\|_{L^{2}}^{\frac{1}{6}}\left\|\nabla^{2} d\right\|_{L^{2}}^{\frac{1}{3}}\left\|\nabla^{5} d\right\|_{L^{2}}^{\frac{2}{3}}\right\}^{2}+\frac{1}{16}\left\|\nabla^{4} u\right\|_{L^{2}}^{2} \\
\leq & C\left(\left\|\nabla^{2} d\right\|_{L^{2}}^{14}+\|\nabla u\|_{L^{2}}^{14}\right)+\frac{1}{8}\left(\left\|\nabla^{4} u\right\|_{L^{2}}^{2}+\left\|\nabla^{5} d\right\|_{L^{2}}^{2}\right),
\end{aligned}
$$

and

$$
\begin{aligned}
\left|I_{3,4}\right| & =\left|\left\langle\partial_{x}^{3}\left(|\nabla d|^{2} d\right), \partial_{x}^{5} d\right\rangle\right| \\
& \leq C\left\|\partial_{x}^{3}\left(|\nabla d|^{2} d\right)\right\|_{L^{2}}^{2}+\frac{1}{32}\left\|\nabla^{5} d\right\|_{L^{2}}^{2} \\
& \leq C\left(\left\|\nabla^{3}|\nabla d|^{2}\right\|_{L^{2}}^{2}+\left\|\left.\nabla d\right|^{2}\right\|_{L^{\infty}}\left\|\nabla^{3} d\right\|_{L^{2}}^{2}\right)+\frac{1}{32}\left\|\nabla^{5} d\right\|_{L^{2}}^{2} \\
& \leq C\left(\|\nabla d\|_{L^{\infty}}^{2}\left\|\nabla^{4} d\right\|_{L^{2}}^{2}+\|\Delta d\|_{L^{\infty}}^{2}\left\|\nabla^{3} d\right\|_{L^{2}}^{2}\right)+\frac{1}{32}\left\|\nabla^{5} d\right\|_{L^{2}}^{2} \\
& \leq C\left(\left\|\nabla^{2} d\right\|_{L^{2}}^{14}+\left\{\left\|\nabla^{2} d\right\|_{L^{\frac{1}{2}}}^{2}\left\|\nabla^{5} d\right\|_{L^{2}}^{\frac{1}{2}}\left\|\nabla^{2} d\right\|_{L^{2}}^{\frac{2}{3}}\left\|\nabla^{5} d\right\|_{L^{2}}^{\frac{1}{3}}\right\}^{2}\right)+\frac{1}{16}\left\|\nabla^{5} d\right\|_{L^{2}}^{2} \\
& \leq C\left\|\nabla^{2} d\right\|_{L^{2}}^{14}+\frac{1}{8}\left\|\nabla^{5} d\right\|_{L^{2}}^{2} .
\end{aligned}
$$

Putting (2.13)-(2.16) into (2.5) for $|\alpha|=3$ and by (2.11), we arrive at

$$
\begin{aligned}
& \frac{d}{d t}\left(\left\|\nabla^{3} u\right\|_{L^{2}}^{2}+\left\|\nabla^{4} d\right\|_{L^{2}}^{2}\right)+\left\|\nabla^{4} u\right\|_{L^{2}}^{2}+\left\|\nabla^{5} d\right\|_{L^{2}}^{2} \\
& \leq C\left(\left\|\nabla^{2} d\right\|_{L^{2}}^{14}+\|\nabla u\|_{L^{2}}^{14}\right) \leq C_{*}(1+H(t))^{C_{0} \varepsilon}
\end{aligned}
$$

for all $T_{*} \leq t<T$. Integrating the above inequality with respect to time from $T_{*}$ to $t \in\left[T_{*}, T\right)$, we obtain

$$
\begin{aligned}
& 1+\left\|\nabla^{3} u(t)\right\|_{L^{2}}^{2}+\left\|\nabla^{4} d(t)\right\|_{L^{2}}^{2} \\
\leq & C\left(1+\left\|\nabla^{3} u\left(T_{*}, \cdot\right)\right\|_{L^{2}}^{2}+\left\|\nabla^{4} d\left(T_{*}, \cdot\right)\right\|_{L^{2}}^{2}+C_{*}(1+H(t))^{C_{0} \varepsilon}\right) .
\end{aligned}
$$

If we choose $\varepsilon$ small such that $C_{0} \varepsilon<\frac{1}{2}$, then (2.17) implies

$$
1+H(t) \leq C\left(1+\left\|\nabla^{3} u\left(T_{*}, \cdot\right)\right\|_{L^{2}}^{2}+\left\|\nabla^{4} d\left(T_{*}, \cdot\right)\right\|_{L^{2}}^{2}\right),
$$

for all $T_{*} \leq t<T$. Since the right hand side of above inequality is independent of $t$ for $T_{*} \leq t<$ 
$T$, we conclude that the above inequality is valid for $t=T$ which means that $u(T) \in H^{3}\left(\mathbb{R}^{3}\right)$ and $d(T)-a \in H^{4}\left(\mathbb{R}^{3}\right)$. Thus the proof of Theorem 1.1 is completed.

\section{References}

\section{References}

[1] J. T. Beale, T. Kato, A. Majda, Remarks on the breakdown of smooth solutions for the 3-D Euler equation. Commun. Math. Phys. 94: 61-66.

[2] P. G. DE Gennes, The Physics of Liquid Crystals. Oxford, 1974.

[3] J. L. Ericksen, Hydrostatic theory of liquid crystal. Arch. Ration. Mech. Anal. 9 (1962), 371-378.

[4] J.S. Fan, J.K. Li, Regularity criteria for the strong solutions to the Ericksen-Leslie system in $\mathbb{R}^{3}$, J. Math. Anal. Appl., 425 (2015), no. 2, 695-703.

[5] M. C. Hong, Global existence of solutions of the simplified Ericksen-Leslie system in $\mathbb{R}^{2}$. Calc. Var. Partial Differential Equations 40 (2011), 15-36.

[6] M. C. Hong, Z. P. Xin, Global existence of solutions of the Liquid Crystal flow for the Oseen-Frank model in $\mathbb{R}^{2}$. Adv. Math., 231 (2012), 1364-1400.

[7] M.C. Hong, J.K. Li, Z.P. Xin, Blow-up criteria of strong solutions to the Ericksen-Leslie system in $\mathbb{R}^{3}$. Comm. Partial Differential Equations, 39 (2014), no. 7, 1284-1328.

[8] T. Huang, C. Y. Wang, Blow up criterion for nematic liquid crystal flows. Comm. Partial Differ. Equ. 37 (2012), 875-884.

[9] T. Huang, F.H. Lin, C. Liu, C.Y. Chang, Blow up criterion for the incompressible nematic liquid crystal flows, arXiv:1504.01080.

[10] H. Kozono, Y. Taniuchi, Bilinear estimates in BMO and the Navier-Stokes equations. Math. Z. 235 (2000), 173-194.

[11] Z. Lei, Y. Zhou, BKM's criterion and global weak solutions for magnetohydrodynamics with zero viscosity, Discrete Contin. Dyn. Syst., 25 (2009), no. 2, 575-583.

[12] Z. Lei, D. Li, X.Y. Zhang, Remarks of global wellposedness of liquid crystal flows and heat flows of harmonic maps in two dimensions. Proc. Amer. Math. Soc., 142 (2014), no. 11, 3801-3810.

[13] F. M. Leslie, Some constitutive equations for liquid crystals. Arch. Ration. Mech. Anal. 28 (1962), $265-283$.

[14] J.K. Li, S.E. Tii, Z.P. Xin, On the uniqueness of weak solutions to the Ericksen-Leslie liquid crystal model in $\mathbb{R}^{2}$, Math. Models Methods Appl. Sci. 26 (2016), no. 4, 803-822.

[15] J.K. Li, Z.P. Xin, Global Weak Solutions to Non-isothermal Nematic Liquid Crystals in 2D, arXiv:1307.2065.

[16] F. H. Lin, Nonlinear theory of defects in nematic liquid crystals; phase transition and flow phenomena. Commun. Pure Appl. Math. 42 (1989), 789-814.

[17] F. H. Lin, C. Liu, Nonparabolic dissipative systems modeling the flow of liquid crystals. Commun. Pure Appl. Math. 48 (1995), 501-537.

[18] F. H. Lin, C. Liu, Partial regularity of the dynamic system modeling the flow of liquid crystals. Discrete Contin. Dyn. Syst. 2 (1996), 1-22.

[19] F. H. Lin, J. Lin, C. Y. Wang, Liquid crystal flows in two dimensions. Arch. Rational Mech. Anal. 197 (2010), 297-336.

[20] F. H. Lin, J. Lin, C. Y. Wang, On the uniqueness of heat flow of harmonic maps and hydrodynamic flow of nematic liquid crystals. Chinese Ann. Math. 31B: 921-928.

[21] F.H. Lin, C.Y. Chang, Global existence of weak solutions of the nematic liquid crystal flow in dimensions three, arXiv:1408.4146.

[22] A. Majda, Compressible fluid flow and system of conservation laws in several space variables. Applied Mathematical Sciences, 53, Springer- Verlag, NewYork, 1984.

[23] X. Xu and Z. Zhang, Global regularity and uniqueness of weak solution for the 2-D liquid crystal flows. J. Differential Equations 252 (2012), 1169-1181. 